\title{
A cooperative construction method for the measurement matrix and sensing dictionary used in compression sensing
}

\author{
Zhi Yuan Shen, Xin Miao Cheng ${ }^{*}$ (D) and Qian Qian Wang
}

\begin{abstract}
A measurement matrix and sensing dictionary are the basic tools for signal compression sampling and reconstruction, respectively, which are important aspects in the field of compression sensing. Previous studies which have divided the measurement matrix and sensing dictionary into two separate processes did not make full use of their inherent intercorrelations. In case of which could be fully utilized, the mutual coherence of the atoms of measurement matrix and sensing dictionary can be further reduced under the premise of ensuring that the original signal information is stored, which could improve the accuracy of signal recovery. The present study attempted to reduce the mutual coherence between the sensing dictionary and measurement matrix by proposing the t-average mutual coherence coefficient as an evaluation index for the sensing dictionary. A mathematical model for co-constructing a measurement matrix and sensing dictionary is firstly proposed. Then, the measurement matrix and sensing dictionary cooperative construction(MSCA)algorithm is proposed to solve the model at a faster rate. The simulated results for sparse signal and binary image show that the proposed algorithm has faster computing speed and higher solution precision than the state-of-the-art construction algorithms.
\end{abstract}

Keyword: Compression sensing, Measurement matrix, Sensing dictionary, Cooperative construction

\section{Introduction}

ADS-B (automatic dependent surveillance and broadcast) is an important technology for monitoring aircraft operations. However, the effects of signal interference on this method can introduce hazards into aircraft operations. One potential way of addressing this problem is to use an array antenna to estimate the DOA (direction of signal arrival) based on the spatial sparsity of ADS-B signals. Implementing this method requires an accurate signal recovery algorithm that is effective in the presence of a high compression ratio. Compression sensing (CS) was first proposed for breaking the limitation of the Nyquist sampling theorem, by permitting the original sparse signal to be reconstructed with high accuracy from a small amount of sampling data using an optimization algorithm.

CS involves constructing a measurement matrix and applying a recovery algorithm to the sparse signal. There has been a considerable amount of researches about the

\footnotetext{
* Correspondence: 459188293@qq.com

College of Civil Aviation, NanJing University of Aeronautics and Astronautics, NanJing 210000, China
}

construction of the measurement matrix, because the information in the original signal preserved during compression sampling plays a vital role in determining the signal recovery performance. Several studies have demonstrated the applicability effectiveness of a Gaussian random matrix [1], Bernoulli random matrix [2], and random local Hadamard matrix as the measurement matrix. However, the application of a random matrix has some drawbacks associated with the presence of random elements, such as the difficulty of implementation in hardware, high storage complexity, and transmission difficulties [3]. These drawbacks mean that using a random matrix cannot ensure the stability and efficiency of compression sampling, which led to the proposal of using a deterministic measurement matrix [4]. The most popular algorithm for constructing deterministic matrices can be divided into four categories based on different generation methods for the matrix elements: (1) a finite framework, such as the expander graph [5]; (2) encoding, such as chirp codes [6] or the Toeplitz deterministic matrix [7]; (3) the maximum Welch boundary, such as 
that constructed using a difference set algorithm [8] and numerical searching algorithm [9]; and (4) a training mode by optimizing the restricted isometry property of the initial matrix [10] and implemented by a projection algorithm and gradient-based training algorithm.

This paper focuses on the fourth category, namely, on an algorithm for constructing the measurement matrix based on training. The reason is that it can exhibit better signalrecovery performance while not being constrained by the matrix dimensions compared with the other methods. By constructing a sensing dictionary that has the same dimensions and a low mutual coherence with the measurement matrix, the sensing dictionary included in the reconstruction algorithm can achieve a better sparse approximation to the original signal. Consequently it improves the accuracy of signal recovery. The previous study demonstrated that a measurement matrix with a smaller mutual coherence can significantly improve the signal recovery performance [11]. This study proposes a measurement matrix and sensing dictionary cooperative construction algorithm (MSCA) to improve the accuracy of the original sparse signal by utilizing a sensing dictionary.

The remainder of this paper is organized as follows. In Section 2, the conventional sensing dictionary method is introduced. In Section 3, a mathematical model for coconstructing a measurement matrix and sensing dictionary is firstly proposed. Then, the measurement matrix and sensing dictionary cooperative construction (MSCA) algorithm is proposed to solve the model. In Section 4, a series of simulations are conducted. In Section 5, conclusions and future research directions are presented.

\section{Sensing dictionary}

For a one-dimensional sparse signal, $x \in R^{N \times 1}$, with a measurement matrix $\Phi \in R^{M \times N}, M \ll N$. The compressed signal can be described as $y=\Phi x, y \in R^{M \times 1}$. The viewpoint of energy loss also means the information loss in the signal during compression sampling. Suppose that $\Phi=I$ and it holds that $\|x\|_{2}^{2}=\|\Phi x\|_{2}^{2}$. This means that the energy of the original signal can be preserved $100 \%$, although this is hardly ever the case for most $\Phi \in R^{M \times N}$ when $M \ll N$. It is obvious that a smaller difference between measurement matrix $\Phi$ and the orthogonal matrix will maintain the energy in the original signal and so improve the ability to perform the reconstruction. On the other hand, from the viewpoint of signal recovery, the state-of-the-art OMP reconstruction algorithm can be described as follows: calculate $b=\Phi^{T} y$ and determine the maximum in vector $b$, whose position corresponds to the position of the atom in $\Phi$ that matches best up to $y$. Suppose that $\Phi$ satisfies $\Phi^{T} \Phi=I$, then $b=\Phi^{T} y=$ $\Phi^{T} \Phi x=x$. A precise signal reconstruction can be obtained in this situation, namely, the identified elements in the supporting set are $100 \%$ accurate. The above assumptions indicate that constructing internal atoms of measurement matrix $\Phi$ requires a low mutual coherence; however, this situation is almost never achieved since $\Phi$ is not a square matrix.

In order to resolve the above difficulties, a sensing dictionary $\Sigma$ that has the same dimensions and a low mutual coherence with $\Phi$ needs to be constructed. $\Sigma^{T} \Phi$ has greater orthogonality than does $\Phi^{T} \Phi$. And then, we use the sensing dictionary to take place of the measurement matrix to reconstruct the signal, since of the coherence between internal atoms of sensing dictionary is lower than measurement matrix, and the sensing dictionary could better save the information of the original signal. As a result, we could achieve a better sparse approximation to the original signal in signal recovery. The elements of the support set are then identified using a greedy algorithm such as the OMP algorithm. This algorithm can be described as follows:

$$
i=\underset{1 \leq i \leq N}{\operatorname{argmax}}\left|\left\langle\varepsilon_{i}, y\right\rangle\right|
$$

where $\varepsilon_{i}$ is the $i$ th atom of sensing dictionary $\Sigma$.

The analysis above shows that the goal of constructing the measurement matrix is to reduce the coherence between the internal atoms. The $t$-average coherence coefficient is defined as $[12,13]$

$$
\mu_{t}(\Phi) \triangleq \frac{\sum_{1 \leq i, j \leq N, i \neq j}(|G(i, j)| \geq t)|G(i, j)|}{\sum_{1 \leq i, j \leq N, i \neq j}(|G(i, j)| \geq t)}
$$

where $G(i, j)$ is the element at the $i$ th row and $j$ th column of the Gram matrix $G=\Phi^{T} \Phi$. The $t$-average coherence coefficient is the average value of all non-diagonal elements with an absolute value greater than a specific threshold $t$ in matrix G. The sensing dictionary is constructed with the aim of $\Sigma^{T} \Phi$ being a sufficiently accurate approximation to $I$. In this situation, the inner product of each corresponding atom in $\Sigma$ and $\Phi$ obey

$$
\left\{\begin{array}{c}
\left|\left\langle\varepsilon_{i}, \phi_{i}\right\rangle\right|=1, \text { if } i=j \\
\left|\left\langle\varepsilon_{i}, \phi_{i}\right\rangle\right|=0, \text { if } i \neq j
\end{array}\right.
$$

The sensing dictionary and measurement matrix were constructed cooperatively in this study based on a revised $t$-average index. The basic idea is to first set up the inner product between $\phi_{i}$ and $\varepsilon_{i}$ equal 1 , according to Eq. (3), and then calculate $G^{\prime}=\sum^{T} \Phi$ and tighten the non-diagonal elements so that they gradually approach 0 using a greedy algorithm until $\mu_{t}(\Sigma, \Phi)$ satisfies threshold $t$. After these steps are finished, a pair of $\Phi$ and $\Sigma$ can be constructed. This process can be described as follows: 


$$
\underset{\Phi, \sum}{\operatorname{argmin}}\left\|\sum^{T} \Phi-I\right\|_{F}^{2}
$$

The value of threshold $t$ can be set to $>0$ in order to reduce the number of iterations. Tropp et al. [14] demonstrated that the minimum achievable value of the non-diagonal elements was $t_{E}=\sqrt[E]{\frac{n-m}{m(n-1)} \pm}$ for a matrix with the ETF (equidimensional tight framework) property. Because $G$ is similar to the ETF matrix, we set $t=$ $t_{E}$. Equation (4) can be modified to

$$
\underset{\Phi, \sum}{\operatorname{argmin}}\left\|\sum^{T} \Phi-H\right\|_{F}^{2}
$$

where matrix $H \in R^{N \times N}$ and has unit diagonal elements and non-diagonal elements equal to $t_{E} \operatorname{sign}\left(G^{\prime}(i\right.$, j)).

\section{Co-construction algorithm}

In order to evaluate the performance of the sensing dictionary, the $t$-average mutual coherence coefficient is proposed:

$$
\mu_{t}(\Sigma, \Phi) \triangleq \frac{\sum_{1 \leq i, j \leq N, i \neq j}\left(\left|G^{\prime}(i, j)\right| \geq t\right)\left|G^{\prime}(i, j)\right|}{\sum_{1 \leq i, j \leq N, i \neq j}\left(\left|G^{\prime}(i, j)\right| \geq t\right)}
$$

where $G^{\prime}(i, j)$ is the $i j$ th element of Gram matrix $G^{\prime}=\Sigma^{T} \Phi . \mu_{t}(\Sigma, \Phi)$ calculates the average value of all non-diagonal elements in $G$ with absolute values greater than the specific threshold $t$. A smaller $\mu_{t}(\Sigma, \Phi)$ will make $\Sigma^{T} \Phi$ closer to $I$ and result in the sensing dictionary exhibiting better performance.

Measurement matrix $\Phi$ and sensing dictionary $\Sigma$ can be obtained by solving Eq. (8), which can be decomposed into the following two subproblems:

$$
\begin{aligned}
& \text { (1) construct } \Phi=\arg \min _{\Phi}\left\|\Phi^{T} \Phi-H\right\|_{F}^{2} \text {; } \\
& \text { (2) construct } \Sigma=\arg \min _{\Sigma}\left\|\Sigma^{T} \Phi-H\right\|_{F}^{2} \text {; }
\end{aligned}
$$

These subproblems can be alternately solved using an iterative process, with the performance of $\Phi$ and $\Sigma$ then evaluated using Eqs. (2) and (6) in each iteration. If the variation between two results of consecutive iterations is less than threshold $\xi$, or the number of iterations exceeds a defined maximum, the algorithm is terminated and $\Phi$ and $\Sigma$ are outputted.

\subsection{Gradient-based approach for constructing the measurement matrix}

A descending gradient-based method was adopted for solving problem (1) in this study that can reduce the value of nondiagonal elements of $G=\Phi^{T} \Phi$ [15], namely, the mutual coherence of the internal atoms in $\Phi$ can be reduced gradually. The optimization routine can be described as follows:

1) Define the cost function as $C=\left\|\Phi^{T} \Phi-H\right\|_{F}^{2}$;

2) Calculate the gradient of the cost function:

$$
\frac{\partial C}{\partial \Phi}=\frac{\partial}{\partial \Phi} \operatorname{Tr}\left\{\left(\Phi^{T} \Phi-H\right)^{T}\left(\Phi^{T} \Phi-H\right)\right\}
$$

Simplify (7) with the matrix derivative rule [6] to

$$
\frac{\partial C}{\partial \Phi}=4 \Phi\left(\Phi^{T} \Phi-H\right)
$$

3) The full update equation will then be

$$
\Phi_{(k+1)}=\Phi_{(k)}-\beta \frac{\partial C}{\partial \Phi_{k}}
$$

where $k$ is the number of iterations and $\beta$ is the step length.

4) Calculate the $t$-average coherence coefficient of $\Phi$ using Eq. (2), and estimate whether the variation between two results of consecutive iterations is less than threshold $\xi$; if so, terminate the algorithm and output $\Phi$.

The above method is used to update $\Phi$ so as to gradually reduce cost function $C$ and finally converge to a local optimal value. The convergence of the above method is proved in $[16,17]$. The paper above proves that the algorithm performs to the maximal iterations and gets the convergency value when $\beta$ takes a minimum of 0.001 . The rate of convergence and the convergency value be expressed by $\mu_{t}(\Phi)$ are equivalent when $0.005 \leq \beta \leq 0.01$, namely, the value of $\beta$ in this range has little effect on the convergence of gradient algorithm.

\subsection{Tightening and SVD-based approach for the sensing dictionary}

Measurement matrix $\Phi$ will be obtained after solving problem (1). Problem (2) can be solved based on the previous results. There are two key points associated with solving problem (2): (1) take full advantage of the correlation between matrices $\Sigma$ and $\Phi$ and (2) ensure that the mutual coherence between $\Sigma$ and $\Phi$, namely $\mu_{t}(\Sigma, \Phi)$, is as low as possible. In order to implement these two points in the process of solving the problem, this study proposed the following methods: (1) construct matrix 
$G^{\prime}=\Sigma^{T} \Phi$, (2) shrink non-diagonal elements of $G^{\prime}$ using the tightening operator to gradually make $G$ 'close to $H$, and (3) obtain a pair of sensing dictionary and measurement matrix by SVD decomposition [18].

The numerical range of non-diagonal elements of matrix $G$ is $[-1,1]$ since $\Phi$ and $\Sigma$ were normalized in the initial stage, and this range could be further reduced to $[-\gamma, \gamma](\gamma<1)$ using a tightening operator. A simple operator for mapping $[-1,1]$ to $[-\gamma, \gamma]$ is $[21]$

$$
\rho=\frac{4}{\pi} \gamma \arctan \left(G^{\prime}(i, j)\right)
$$

The tightening operator only needs one parameter to adjust the scope of non-diagonal elements of $G$ in the iteration process; we set $\gamma=0.4$ in this study.

Finally, matrix $G$ is decomposed by SVD to achieve a pair of $\Phi$ and $\Sigma$ [19]:

$$
G^{\prime}=U^{T} V W
$$

In order to make $G$ closer to $H$, we arranged all diagonal elements in $V$ from the top left to bottom right and retained the maximum $M$ elements in a new matrix $V_{M}$ [20]. $\Phi$ and $\Sigma$ could then be constructed as follows:

$$
\sum=V_{M}^{\frac{1}{2}} U, \Phi=V_{M}^{\frac{1}{2}} W
$$

In order to ensure the inner-product corresponding atoms between $\Phi$ and $\Sigma$ equal 1, the following equations should be used:

$$
\phi_{i}=\frac{\phi_{i}}{\left\|\phi_{i}\right\|_{2}}, \quad \varepsilon_{i}=\frac{\varepsilon_{i}}{\left|\left\langle\varepsilon_{i}, \phi_{i}\right\rangle\right|}
$$

This approach constructs a pair of sensing dictionary $\Sigma$ and measurement matrix $\Phi$ with low mutual coherence. The MSCA is listed in Algorithm 1.

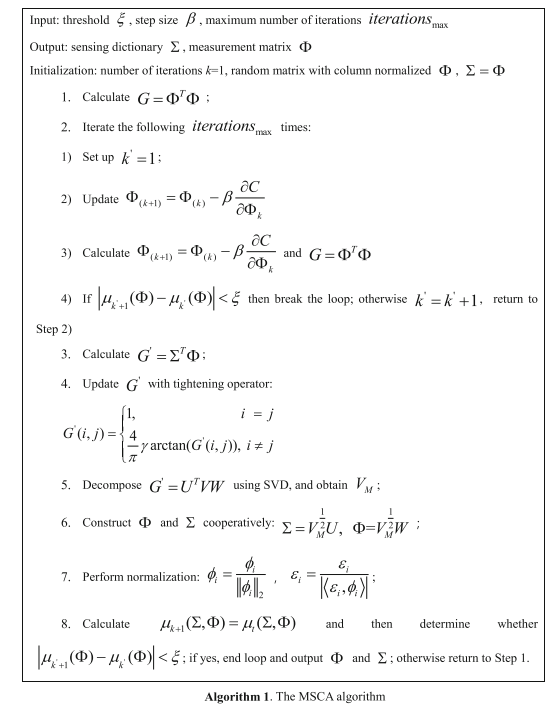

3.2.0.1 Algorithm 1. The MSCA algorithm

\section{Simulations and discussion}

We used simulations to verify the validity of the MSCA. Suppose that the length of the original sparse signal is $N$ $=256$, the measurement matrix is $\Phi$, and the sensing dictionary is $\Sigma$ with $\Phi, \Sigma \in R^{M \times N}$, where $M$ is the number of measurements. In order to access the performance of matrix $\Phi$ or $\Sigma$ for different compression ratios, we varied $M$ from 100 to 150 with a step size of 5 . We adopted the matrix coherence coefficient and $t$-average mutual coherence coefficient as the evaluation indices, and the OMP algorithm was used for signal reconstruction. In addition, a Gaussian random matrix and gradient algorithm [21] were selected for comparison. The corresponding Welch boundary was used to compare the performances of the various algorithms. The simulation results are shown in Fig. 1.

Figure 1 indicates that the MSCA can reduce the coherence of the matrix compared with the Gaussian random matrix and the gradient algorithm. Figure 1a shows that both the MSCA and the gradient algorithm are more effective than the Gaussian random matrix in reducing the coherence of $\Phi$, and the performance of the MSCA is slightly better than that of the gradient algorithm: based on the gradient algorithm, the coherence coefficient of the matrix decreased gradually from 0.22 to 0.17 as the measured signal length changed, while the index decreased gradually from 0.11 to 0.08 based on the MSCA, which is closer to the Welch boundary. Figure $1 \mathrm{~b}$ presents the results for the $t$-average coherence coefficient as an index to evaluate the performance of $\Phi$ between the various algorithms. The MSCA was still closer to the Welch boundary than for the Gaussian random matrix and the gradient algorithm. Figure 1c shows the performance of the sensing dictionary when comparing the $t$-average mutual coherence coefficient of $\Sigma$ and the $t$-average coherence coefficient of $\Phi$. The figure indicates that for the same signal compression ratio, the $t$-average mutual coherence coefficient of $\sum$ was smaller than the $t$-average coherence coefficient of $\Phi$. Namely, the average of the off-diagonal elements of $G^{\prime}=\Sigma^{T} \Phi$ is less than the $G=\Phi^{T} \Phi$, which means that $\Sigma^{T} \Phi$ approaches $I$ more closely on the premise of diagonal elements equal 1 [22, 23]. Collectively, the $t$-average mutual coherence coefficient of the sensing dictionary could be closer to the Welch boundary, so that the original signal support set can be identified more accurately through $\Sigma$ [24].

We compared the reconstruction performances of the abovementioned algorithms. It is clear from Fig. 2 that the reconstruction performances of the gradient algorithm and the MSCA were better than that of the Gaussian random matrix. In Fig 2a, some signal components are not recovered because of the internal atoms of the Gaussian random matrix have bigger mutual coherence than other two matrix [25]. 


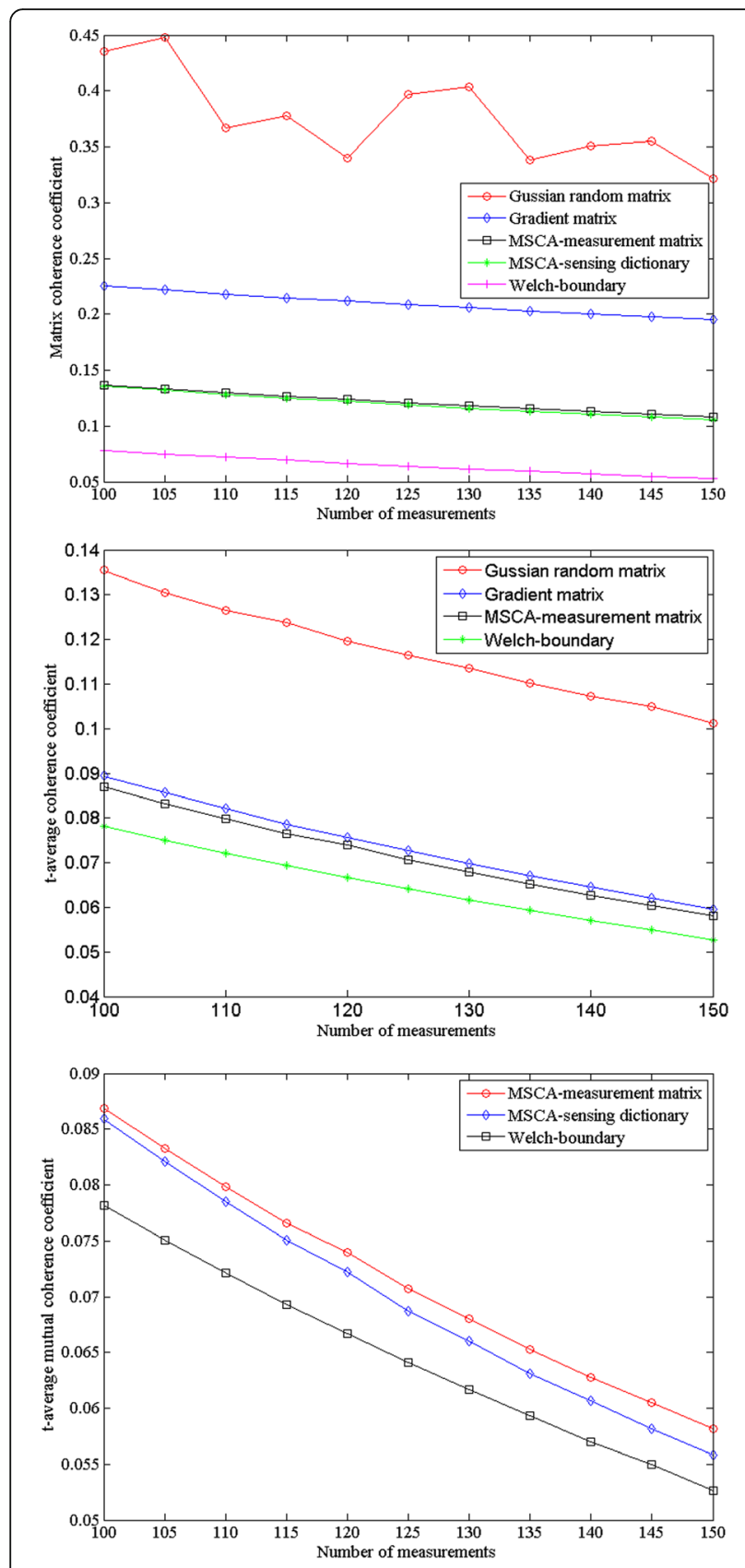

Fig. 1 Performances of the measurement matrices constructed using various algorithms. a Matrix coherence coefficient. $\mathbf{b} t$-average coherence coefficient. c $t$-average mutual coherence coefficient

In order to accurately estimate the precision of the recovery result, we calculated the reconstruction error as

$$
\text { error_reconstruction }=\frac{\|x-\widehat{x}\|_{2}}{\|x\|_{2}}
$$

We remain the size of the original signal unchanged in the simulation and set the signal sparsity as a variable.

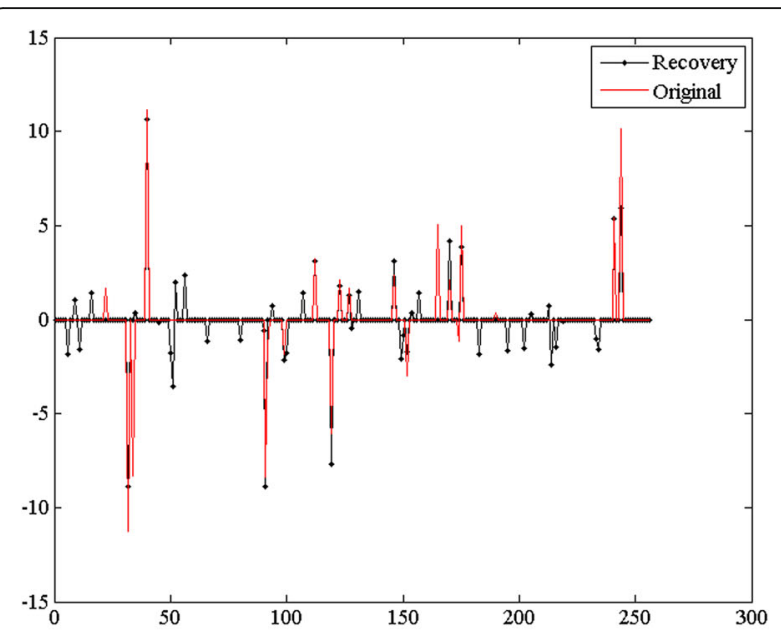

(a) Gaussian random matrix

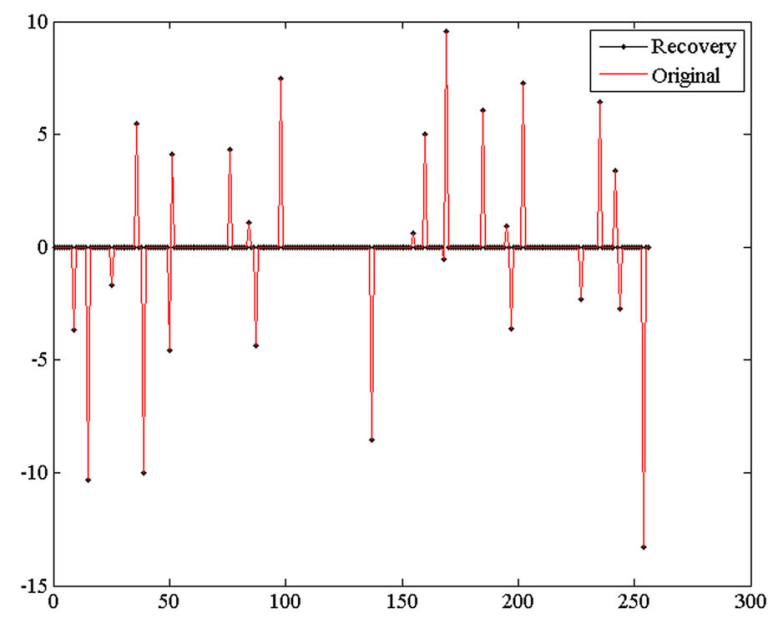

(b) Gradient algorithm

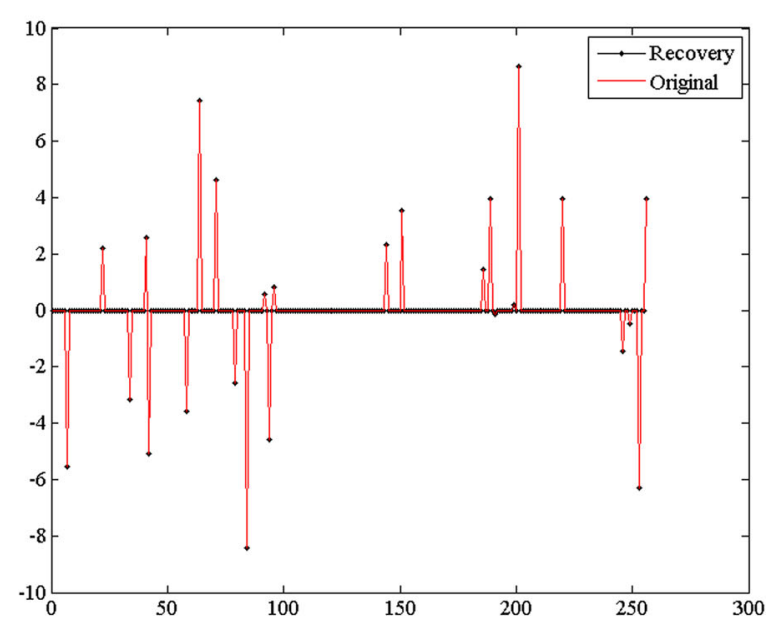

(c) MSCA

Fig. 2 Performance in reconstructing sparse sampled signals for various algorithms. a Gaussian random matrix. b Gradient algorithm. c MSCA 
Signal sparsity indicates the number of non-zero bits in the signal. The results show the signal reconstruction under different signal sparsity and with competing measurement dictionary methods, namely Gaussian random matrix and the gradient matrix [26]. Since the original constructed sparse signal is random, the simulation was repeated 100 times with different signal sparsity, and the average error was calculated. The results are presented in Fig. 3.

We construct a pair of measurement matrix and sensing by the use MSCA algorithm in this simulation; the MSCA measurement matrix in Fig. 3 indicates the average reconstruction error based on MSCA measurement matrix only. Correspondingly, the MSCA cooperative construction in Fig. 3 indicates the average reconstruction error based on measurement matrix and MSCA sensing dictionary cooperatively. While, we indicate the MSCA sensing dictionary to show the result obtained reconstructing the signal with the measurement matrix lonely. Figure 3 shows that the reconstruction error was smallest for the MSCAcooperative construction in the same signal sparsity, followed by the MSCA-measurement matrix and gradient algorithm, and highest for the Gaussian random matrix. Moreover, the MSCA was better than the Gaussian random matrix in terms of reconstruction stability. Result of simulation proves that the sensing dictionary plays a better performance in reconstruction.

In order to verify the processing ability of the MSCA algorithm to the image, we use the MSCA matrix to compress an image like Fig. 4a. By the way, this paper only simulates the binary image for the purpose of simplifying the process of compression and reconstruction [27, 28]. The stimulation experimental flow is as follows: (1) converting the original image to a 0 1 matrix $r$ with $r \in R^{M \times N}$ by binarization; (2) $N$ pair of measurement matrix $\Phi_{i}$ and sensing dictionary $\sum_{i}$ with $\Phi_{i}, \quad \sum_{i} \in R^{m \times M}, \quad m<M$ be constructed by using MSCA algorithm; (3) compressive sampling: $Y=\Phi Y$, $y_{i}=\Phi_{i} Y_{i}, i=1,2, \ldots, N$; (4) the signal $\gamma_{\text {is }}$ reconstructed by using the sensing dictionary-based OMP algorithm [29]; and (5) image reconstruction and the result are shown as the Fig. 4c.

It is observed that the results of image reconstruction by MSCA sensing dictionary and gradient matrix are better than the Gaussian random matrix by comparing Fig. 4 a, b, c, and d. In order to compare the effects of compression sensing of the three methods on the image signal more clearly, we calculate the reconstruction error as formula (14). The results are shown in the Table 1. Specific results are as follows:

This illustrates that the MSCA matrix could retain the information of the original signal better than the Gaussian random matrix and gradient matrix, and the coupled sensing dictionary also could be used in the reconstruction for signal [30, 31].

\section{Conclusion}

This study addressed the optimization of compression measurements. In this work, a mathematical model for co-constructing a measurement matrix and sensing dictionary was proposed. Then, the measurement matrix and sensing dictionary cooperative

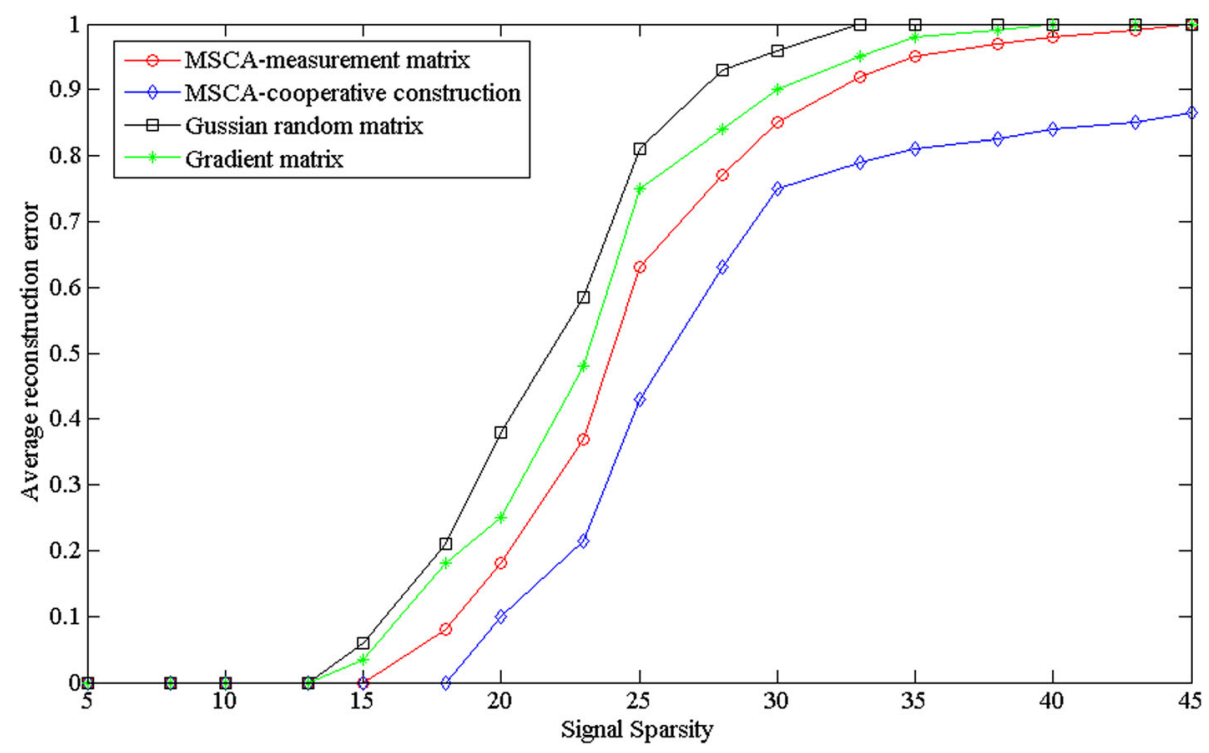

Fig. 3 Average reconstruction error 


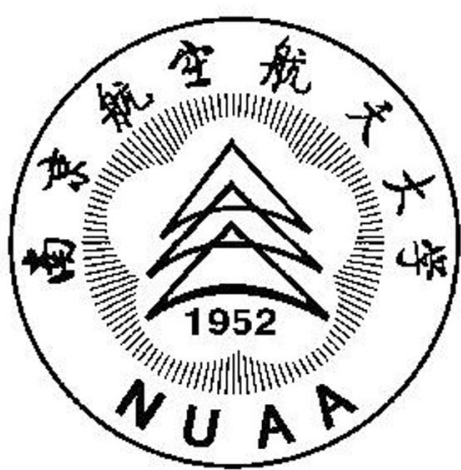

(a) Original Image

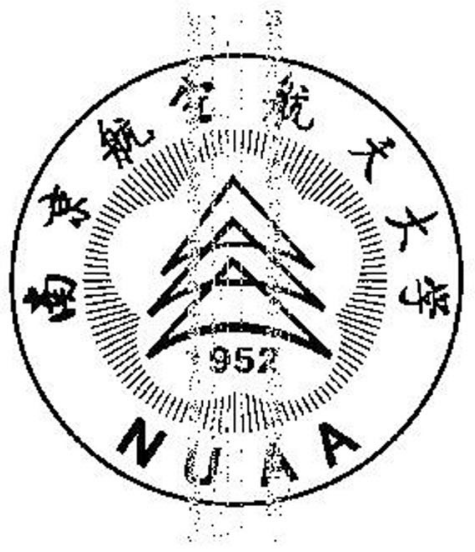

(c) Reconstructed Image by Gradient matrix

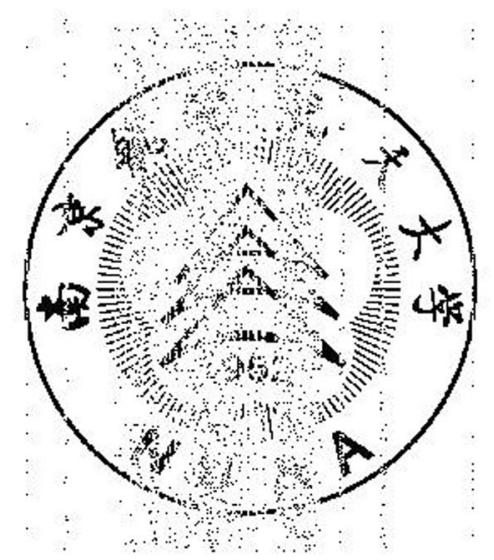

(b) Reconstructed Image by Gussian matrix

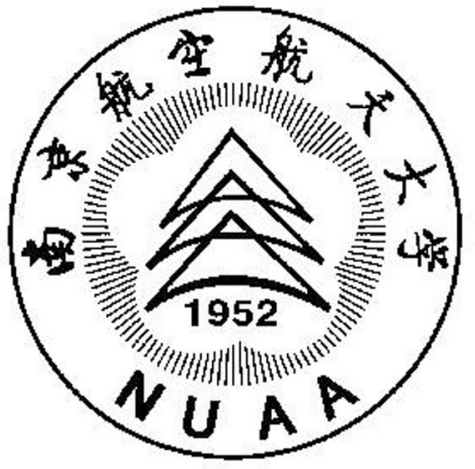

(d) Reconstructed Image by MCSA sensing dictionary

Fig. 4 Compression sensing for image. a Original image. b Reconstructed image by Gaussian matrix. c Reconstructed image by gradient matrix. $\mathbf{d}$ Reconstructed image by MSCA sensing dictionary

construction (MSCA) algorithm is proposed to solve the model at a faster rate. The proposed MSCA makes full use of the inherent correlation between the measurement matrix and sensing dictionary. The advantage of our approach is that it involves constructing the measurement matrix and sensing dictionary cooperatively rather than dividing this into two separate processes, which should also improve the performance in sparse recovery. The $t$-average mutual coherence coefficient was defined for evaluating the performance of the sensing dictionary. The results obtained from simulations have shown that the sensing dictionary has a lower mutual coherence and can successfully reconstruct the sparse signal using the proposed algorithm. Compared with the state-of-art algorithms, the simulation results showed that MSCA is better than the other algorithms in ensuring the low coherence of the measurement matrix and that $\Sigma$ has better coherence than $\Phi$.

Based on the results of the proposed cooperative construction method for the measurement matrix and sensing dictionary in this work, we will continue the following work: (1) sparse reconstructed algorithm based on sensing dictionary [32] and (2) adaptive sparse representation compatible with sensing dictionary [33].

Table 1 The accuracy of reconstruction by the three methods

\begin{tabular}{llll}
\hline Method & Gaussian random matrix & Gradient matrix & MSCA sensing dictionary \\
\hline Accuracy & $74.67 \%$ & $89.43 \%$ & $94.27 \%$ \\
\hline
\end{tabular}




\section{Abbreviations}

ADS-B: Automatic dependent surveillance and broadcast; CS: Compression sensing; DOA: Direction of signal arrival

\section{Acknowledgements}

The authors would like to thank the editorial board and anonymous reviewers for their careful reading and constructive comments which provided important guidance for our paper writing and research work.

\section{Authors' contributions}

ZS determines the research direction of the paper and studies the basic implementation of signal compression sampling. XC developed and implemented the core concepts of the algorithm presented in this manuscript. QW provided refinements and performed data acquisition and generation as well as further supplemental programming. All authors had a significant contribution to the development of early ideas and the design of the final methods. All authors read and approved the final manuscript.

\section{Authors' information}

ZS has a Ph.D. in Control Engineering; has a background in digital signal processing and machine learning; specializes in stochastic processes, stochastic filtering, and machine learning; and is currently working as a senior researcher at the German Research Center for Artificial Intelligence. XC and QW have a Master's degree in Traffic Engineering and specialize in digital signal processing.

\section{Funding}

The authors would like to thank the financial support by China Postdoctoral Science Foundation funded project (2017T100366 and 2016 M601806)

\section{Competing interests}

The authors declare that they have no competing interests.

Received: 28 May 2019 Accepted: 6 January 2020

Published online: 04 March 2020

\section{References}

1. D.L. Donoho, Compressed sensing[J]. IEEE Transactions on information theory 52(4), 1289-1306 (2006)

2. Candès E J. Compressive sampling[J]. Marta Sanz Solé, 2007, 17(2):págs. 1433-1452.

3. Q. Wang, J. Li, Y. Shen, A survey of deterministic measurement matrix construction algorithms in compressed sensing [J]. Acta Electronica Sinica 41(10), 2041-2050 (2013)

4. R.A. Devore, Deterministic constructions of compressed sensing matrices $[M]$. Academic Press, Inc. 2007.

5. F. Li, S. Hong, Y. Gu, et al., An optimization-oriented algorithm for sparse signal reconstruction[J]. IEEE Signal Processing Letters, 2019, PP(99):1-1.

6. L. Applebaum, S.D. Howard, S. Searle, et al., Chirp sensing codes: deterministic compressed sensing measurements for fast recovery[]]. Applied \& Computational Harmonic Analysis 26(2), 283-290 (2009)

7. H. Rauhut, Circulant and Toeplitz matrices in compressed sensing[J]. Mathematics, 2009.

8. C. Ding, Complex codebooks from combinatorial designs[]]. IEEE Transactions on Information Theory It 52(9), 4229-4235 (2006)

9. Q. Liu, Q. Zhang, L. Ying, et al., A fast algorithm for sparse signal reconstruction based on off-grid model[]]. let Radar Sonar Navigation, 2018, 12(4).

10. EJ. Candès, The restricted isometry property and its implications for compressed sensing[]]. Comptes Rendus Mathematique 346(9-10), 589-592 (2008)

11. J.A. Tropp, A.C. Gilbert, Signal recovery from random measurements via orthogonal matching pursuit[]]. IEEE Transactions on Information Theory 53(12), 4655-4666 (2007)

12. S.F. Cotter, B.D. Rao, K. Engan, et al., Sparse solutions to linear inverse problems with multiple measurement vectors[]]. IEEE Transactions on Signal Processing 53(7), 2477-2488 (2005)

13. R. Baraniuk, M. Davenport, R. Devore, et al., A simple proof of the restricted isometry property for random matrices[]]. Constructive Approximation 28(3), 253-263 (2008)

14. J.A. Tropp, I.S. Dhillon, R.W. Heath, et al., Designing structured tight frames via an alternating projection method[]]. IEEE Transactions on Information Theory 51(1), 188-209 (2005)
15. V. Abolghasemi, S. Ferdowsi, S. Sanei, A gradient-based alternating minimization approach for optimization of the measurement matrix in compressive sensing[J]. Signal Processing 92(4), 999-1009 (2012)

16. J. Li, Research on deterministic matrix and sparse recovery algorithm with application to WCSS[D]. Haerbin Institute of Technology, 2016

17. J. Li, Q. Wang, W. Yan, et al., Efficient cooperative compressive spectrum sensing by identifying multi-candidate and exploiting deterministic matrix[]]. EURASIP Journal on Advances in Signal Processing 2015(1), 85 (2015)

18. L.C. Jiao, J. Chen, J. Wu, et al., Efficient collaborative spectrum sensing with low sample rate[J]. Wireless Personal Communications 67(4), 923-936 (2012)

19. K. Schnass, P. Vandergheynst, Dictionary preconditioning for greedy algorithms[J]. IEEE Transactions on Signal Processing 56(5), 1994-2002 (2008)

20. Y. Jiang, H. Wang, R. Shao, et al., Infrared image reconstruction based on archimedes spiral measurement matrix[]]. Journal of Shanghai Jiaotong University (Science) 24(2), 204-208 (2019)

21. D.J. Love, R.W. Heath, T. Strohmer, Grassmannian beamforming for multipleinput multiple-output wireless systems[]]. IEEE Transactions on Information Theory It 49(10), 2735-2747 (2003)

22. A. Huang, G. Guan, Q Wan, et al., A re-weighted algorithm for designing data dependent sensing dictionary[]]. 2011.

23. M. Elad, Optimized projections for compressed sensing[]]. IEEE Transactions on Signal Processing 55(12), 5695-5702 (2007)

24. S. Jafarpour, W. Xu, B. Hassibi, et al., Efficient and robust compressed sensing using optimized expander graphs[]]. IEEE Transactions on Information Theory 55(9), 4299-4308 (2009)

25. K.B. Petersen, M.S. Pedersen, The matrix cookbook[J]. Technical University of Denmark 7, 15 (2008)

26. P. Wang, F. You, S. He, An improved signal reconstruction of modulated wideband converter using a sensing matrix built upon synchronized modulated signals[]]. Circuits, Systems, and Signal Processing 38(7), 31873210 (2019)

27. N. Koep, A. Behboodi, R. Mathar, The restricted isometry property of block diagonal matrices for group-sparse signal recovery[]] (2019)

28. B. Li, Y. Shen, J. Li, Dictionaries construction using alternating projection method in compressive sensing[J]. IEEE Signal Processing Letters 18(11), 663-666 (2011)

29. S. Zhu, C. Zhu, A new image compression-encryption scheme based on compressive sensing and cyclic shift[]]. Multimedia Tools and Applications, 2019(2):1-21.

30. J.A. Tropp, Greed is good: algorithmic results for sparse approximation[J]. IEEE Transactions on Information Theory 50(10), 2231-2242 (2004)

31. C. Ding, T. Feng, A generic construction of complex codebooks meeting the Welch bound[]]. IEEE Transactions on Information Theory 53(11), 42454250 (2007)

32. D.J. Love, R.W Heath, T. Strohmer, Grassmannian beamforming for multipleinput multiple-output wireless systems[]]. IEEE Trans.inform.theory, 2003, 49(10):2735-2747.

33. M.E. Erkoc, N. Karaboga, Evolutionary algorithms for sparse signal reconstruction[J]. Signal, Image and Video Processing, 2019:1-9.

\section{Publisher's Note}

Springer Nature remains neutral with regard to jurisdictional claims in published maps and institutional affiliations. 